2005-06

\title{
Action comprehension: deriving spatial and functional relations.
}

\author{
Bach, $\mathrm{P}$
}

http://hdl.handle.net/10026.1/1023

10.1037/0096-1523.31.3.465

J Exp Psychol Hum Percept Perform

All content in PEARL is protected by copyright law. Author manuscripts are made available in accordance with publisher policies. Please cite only the published version using the details provided on the item record or document. In the absence of an open licence (e.g. Creative Commons), permissions for further reuse of content should be sought from the publisher or author. 


\title{
Action Comprehension: Deriving Spatial and Functional Relations
}

\author{
Patric Bach \\ Max Planck Institute for Psychological Research \\ and University of Wales
}

Thomas C. Gunter and Angela D. Friederici
Max Planck Institute for Cognitive Neuroscience

\author{
Günther Knoblich \\ Max Planck Institute for Psychological Research \\ and Rutgers University \\ Wolfgang Prinz \\ Max Planck Institute for Psychological Research
}

\begin{abstract}
A perceived action can be understood only when information about the action carried out and the objects used are taken into account. It was investigated how spatial and functional information contributes to establishing these relations. Participants observed static frames showing a hand wielding an instrument and a potential target object of the action. The 2 elements could either match or mismatch, spatially or functionally. Participants were required to judge only 1 of the 2 relations while ignoring the other. Both irrelevant spatial and functional mismatches affected judgments of the relevant relation. Moreover, the functional relation provided a context for the judgment of the spatial relation but not vice versa. The results are discussed in respect to recent accounts of action understanding.
\end{abstract}

Keywords: action comprehension, action recognition, mirror neurons, functional knowledge, tool use

Actions of tool use are combinatorial (e.g., Nowak, Plotkin, \& Jansen, 2000), that is, the same action (e.g., inserting something) will have different effects depending on the instruments and target objects used in its course (e.g., canceling a ticket and using a credit card). Thus, theories assuming that actions are understood as soon as their effects are known must address two questions. First, how is the type of action identified? And, second, how do the objects used in its course shape the understanding of the action?

Considerable research has been conducted on the question of how the type of action is identified. It has been proposed that perceived actions were matched directly to the representations an observer would rely on if he or she carried out the actions (e.g., Hommel, Müsseler, Aschersleben, \& Prinz, 2001; Iacoboni, 2005; Knoblich \& Flach, 2003; Prinz, 2005; Rizzolatti, Fogassi, \& Gallese, 2001). Evidence for this idea comes from behavioral studies that demonstrate interference effects between observed and to-beproduced actions (e.g., Brass, Bekkering, Wohlschläger, \& Prinz, 2000; Kilner, Paulignan, \& Blakemore, 2003; Stürmer, Aschersleben, \& Prinz, 2000). Moreover, in the parietal and the premotor cortices, neurons with mirror properties have been discovered that fire when a particular object-directed action is carried out but also

Patric Bach, Max Planck Institute for Psychological Research, Munich, Germany and Centre for Cognitive Neuroscience, University of Wales, Bangor, United Kingdom; Günther Knoblich, Max Planck Institute for Psychological Research and Department of Psychology, Rutgers University; Thomas C. Gunter and Angela D. Friederici, Max Planck Institute for Cognitive Neuroscience, Leipzig, Germany; Wolfgang Prinz, Max Planck Institute for Psychological Research.

We thank Alexander Borkovski, Marco Petz, Stefanie Schuch, and Mike Tucker for their valuable comments and Jana Hiller and Ina Fabbender for the data collection.

Correspondence concerning this article should be addressed to Patric Bach, Centre for Cognitive Neuroscience, University of Wales, Bangor, Gwynedd LL57 2DG, United Kingdom. E-mail: p.bach@bangor.ac.uk when this action is only perceived (DiPellegrino, Fadiga, Fogassi, Gallese, \& Rizzolatti, 1992; Gallese, Fadiga, Fogassi, \& Rizzolatti, 1996; Rizzolatti, Fadiga, Gallese, \& Fogassi, 1996).

These common action representations seem to be coded predominantly in spatial terms. In action production, the parietal lobe relates an object's spatial properties to those of an effector (Milner \& Goodale, 1995; Rossetti \& Pisella, 2002; Ungerleider \& Mishkin, 1982). The object's position is translated into the amplitude and direction of a reaching movement (Bremmer, Duhamel, \& Ben Hamed, 1996), and its orientation, surface features, and size are translated into possible grasp configurations (Murata, Gallese, Luppino, Kaseda, \& Sakata, 2000; Sakata \& Taira, 1994). The premotor cortex then combines these different aspects into a complete action (e.g., Jeannerod, Arbib, Rizzolatti, \& Sakata, 1995; Rizzolatti, Luppino, \& Matelli, 1998). Converging evidence comes from behavioral studies showing that actions are afforded by the spatial features of objects (Tucker \& Ellis, 1998, 2001; Humphreys \& Riddoch, 2001) and that the performing of actions facilitates the detection of spatially suitable target objects (Craighero, Fadiga, Rizzolatti, \& Umiltà, 1999).

During action perception, the parietal areas are also particularly activated when a spatial analysis of the actions is required, for example, when the actions are not known or have to be imitated later (e.g., Decety \& Chaminade, 2003; Decety et al., 1997; Meltzoff \& Decety, 2003). Moreover, they are activated by the perception of object-directed actions in particular and in a somatotopic manner in respect to the effector carrying out the action (Buccino et al., 2001; Manthey, Schubotz, \& von Cramon, 2003). Thus, Oztop and Arbib (2002) have suggested that the premotor cortex guides action by realizing the specific spatial relations between effectors and target objects that are required for the action to succeed. In action perception, the same representations could be used to identify an action from the spatial relations it produces.

Note, however, that the parietal cortex is oblivious to object identity and function (e.g., Milner \& Goodale, 1995), and the firing 
pattern of the mirror neurons does not change when meaningless 3-D solids are acted upon (e.g., Rizzolatti, Fogassi, \& Gallese, 2000). Therefore, actions involving tool use cannot be fully understood by relying solely on the spatial action representations in the parietal-premotor pathway. Rather, the action representations mediating the understanding of such actions also need to incorporate the objects that have to be used. The processing of object identity and function has been associated with areas in the temporal lobe (for a review, see Martin \& Chao, 2001) that receive input from the ventral stream. This pathway has been described as being parallel to the premotor-parietal pathway and has been implicated in selecting objects for action according to the actor's intention (e.g., Milner \& Goodale, 1995). Consistent with this idea, patients with semantic dementia, whose temporal lobe is severely damaged, can neither select another tool with a similar function, nor can they select a typical target object of a tool (Hodges, Spatt, \& Patterson, 1999). Knowledge about object function can be selectively impaired. Ochipa and colleagues (Ochipa, Rothi, \& Heilman, 1989) reported a patient who was able to perform the appropriate movements of everyday tasks but failed to use the appropriate objects (e.g., brushing teeth with a comb). Functional imaging studies revealed temporal lobe activation during action perception, particularly for the observation of meaningful objectdirected actions (Decety et al., 1997). Converging evidence comes from an electrophysiological study in which movies of everyday actions were presented (Sitnikova, Kuperberg, \& Holcomb, 2003). When an instrument with an inappropriate function was chosen (e.g., using a rolling pin instead of a razor for shaving), the N400 component was elicited. The N400 is widely accepted as a measure of semantic integration difficulty (for a review, see Kutas \& Federmeier, 2000), and it originates partially from generators in the temporal lobe (Johnson \& Hamm, 2000; Nobre \& McCarthy, 1995).

We propose that the parietal-premotor and temporal activations during action perception reflect the accessing of distributed action representations that specify (a) which actions have to be carried out for an intended effect to be achieved but also (b) the objects to be used. Thus, an observed action can be understood-and its effect can be derived-if the objects used and the action carried out match such an action representation of the observer. The present study tested one implication of this model. If action comprehension is mediated by representations as described above, observers should not be able to selectively take either objectrelated or action-related information into account, even if the respective other type of information is completely irrelevant to the task and has to be ignored. To test this assumption, we conducted four experiments in which participants observed actions involving the use of a tool. These actions had been rated with regard to (a) the reciprocal associations between instruments and target objects, (b) the familiarity of the actions, and (c) the saliency of the action goal to be achieved (see the Appendix). Ratings were collected in order for us to assess potential influences of these three factors The stimuli were designed in a way that allowed us to independently manipulate the appropriateness of the objects and the appropriateness of the action. The appropriateness of the action was manipulated by varying the spatial relation between instrument and target object. The appropriateness of the objects was manipulated by choosing combinations of instrument and target object that were or were not appropriate to produce a meaningful effect in the environment (e.g., ticket, ticket canceller vs. ticket, credit card reader). Across experiments, participants judged either the appropriateness of the action and were asked to ignore the appropriateness of the objects (Experiments $1 \mathrm{~A}$ and $1 \mathrm{~B})$ or vice versa (Experiments $2 \mathrm{~A}$ and $2 \mathrm{~B}$ ). We hypothesized that if participants solve these tasks by relying on action representations that incorporate object-related and action-related information, they should not be able to refrain from taking either type of irrelevant information into account even when instructed to ignore it.

We were also interested in the question of whether instruments have a special status in action comprehension. Instruments have well-known functions and prototypical target objects. Also, they are applied to these objects in a certain way. Therefore, it is possible that the action to be carried out and the expected-target objects are specified as soon as the instrument is known, but not vice versa. To test this assumption, we presented instrument and target object in different orders. The instrument was presented either before or after the target object. We hypothesized that if the relations are derived from instrument knowledge, irrelevant spatial or functional mismatches should have stronger effects when the instrument is presented before the target object.

\section{Experiment 1A: Plugs}

The first experiment assessed whether participants can ignore functional information about objects when judging whether a perceived action is spatially appropriate. Participants observed two consecutive still frames, one displaying a hand wielding an instrument and one displaying the target object. Still frames of actions were used because they evoke the corresponding actions and motions (e.g., Kourtzi \& Kanwisher, 2000; Stürmer et al., 2000) but also allow reaction times (RTs) to be measured relative to unambiguous stimulus onsets. All actions were plugging actions. For a plugging action to succeed, both the instrument and the slot of the target object should have identical orientations. With orthogonal orientations, a plugging action will not be successful (cf. Figures $1 \mathrm{~A}$ and $1 \mathrm{~B}$ ). In regard to the functional relation, there are certain combinations of plug and target objects that are associated with an effect on the environment (e.g., screwdriver, screw), but others that are not associated with such an effect (e.g., screwdriver, keyhole; cf. Figures 1A and 1C). Thus, there were four conditions: full match (cf. Figure 1A), spatial mismatch (c.f. Figure 1B),
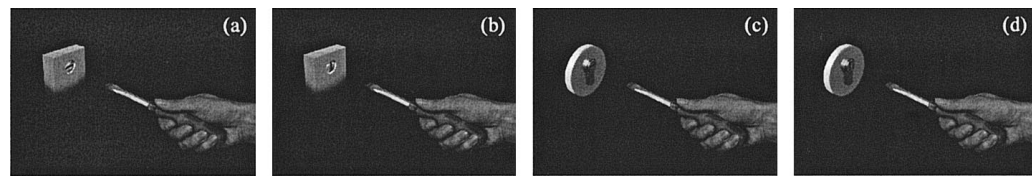

Figure 1. Example of a plugging action: (a) full match, (b) spatial mismatch, (c) functional mismatch, and (d) double mismatch. 
functional mismatch (cf. Figure 1C), and double mismatch (cf. Figure 1D).

The participants' task was to judge whether the spatial relation was appropriate for a plugging action but to ignore the functional relatedness of the objects. Thus, full matches and functional mismatches had to be judged as matching, whereas spatial mismatches and double mismatches had to be judged as mismatching. If observers judge actions relying on action representations that include knowledge about both how an action is carried out and which types of objects are required, then the judgment should be facilitated when the functional relation is appropriate. This is because, for functionally related objects, there should be a corresponding action representation that supplies additional evidence as to the action carried out with them, whereas for functionally unrelated objects no such representation should exist. Note that in the present experiment the functional relation was fully task irrelevant. The stimuli were designed in such a way that the object's parts relevant to the spatial task (i.e., the tip of the instrument and the slot of the target object) were always presented at the same spatial positions. Therefore, participants did not need to identify the objects to find these parts. Moreover, even if instrument and target object were not functionally related, each of them still afforded a plugging action (e.g., ticket, credit card reader).

To test whether instruments and target objects have an asymmetric influence on the activation of the action representations, we varied the order in which the effector or instrument and the object were presented between participants. If instruments are more efficient in accessing the respective action representations, then the effects of irrelevant functional mismatches should be enhanced when presented in the first frame because the required functional relation can then be prespecified. If, however, the functional relation is derived after instrument and target object are known, no differences between the two orders of presentation are expected.

\section{Method}

Participants. Thirty-two participants (16 men, 16 women), all students at the University of Munich, took part in the experiment. They ranged in age from 20 to 35 years. All participants were right-handed and had normal or corrected-to-normal vision. They received a payment of $€ 5$ (\$5) for their participation. Half of the participants were presented with actions preceding the target objects, and the other half used the reverse assignment. The key assignment was counterbalanced across participants. Half of them pressed the left key for a correct judgment and the right key for an incorrect judgment. The other half used the reversed assignment

Materials and apparatus. The stimulus set consisted of 32 photographs. They obtained 9 visual degrees vertically and $6^{\circ}$ horizontally, given the viewing distance of $60 \mathrm{~cm}$. Sixteen pictures showed a hand holding one of the eight different objects to be plugged (screwdriver, coin, key, trolley pin, safety belt, knife, ticket, or credit card) in two different orientations each (vertically or horizontally). The other half of the pictures showed the corresponding eight target objects with the respective openings (screw, slot for the coin, lock, trolley, credit card reader, ticket canceller, knife holder, or slot for safety belt), again, in both orientations.

Out of these photographs, two-frame sequences were assembled. For each stimulus depicting a target object, there were four combinations with different objects to be inserted. One combination created a match on both dimensions so that the device to be inserted and the target opening matched functionally and spatially. One combination created a spatial mismatch, and the functional match was preserved. One combination created a functional mismatch, and the spatial relation remained intact. The last combination created a mismatch for both relations. The resulting stimulus matrix was completely balanced. Any target object or plug, which created one of the mismatches in combination with another stimulus, was completely appropriate in another one. Also, great care was taken that all of the items were photographed under identical lighting conditions and that the slots and pins of functionally mismatching objects were of appropriate size for a plugging action to be carried out. Thus, effects of stimulus differences cannot be made accountable for any differences obtained (see Table 1 for the different combinations of a ticket to the underground, a credit card, and the respective target objects). All in all, there were 64 different two-frame sequences.

For all of these sequences, ratings of (a) the familiarity of the actions, (b) the strength of the associations between instruments and target objects, and (c) the salience of the action effect were obtained (see the Appendix).

Stimulus presentation and data collection were controlled by an Apple Power PC. The pictures were presented on an Apple 21 in. monitor (resolution $1024 \times 768$ pixels). Button presses were recorded from the computer keyboard.

Procedure and design. Upon entering the lab, participants received a computer-based, written instruction. They were told that they would see one object being applied to another object and that their task was to judge whether the orientations of the instrument and the slot of the target object were appropriate for a plugging action. They were also told to ignore whether the two objects usually were used together. The experiment proper lasted for about $30 \mathrm{~min}$ and consisted of 2 blocks of 192 trials each. The order of sequences in each block was randomized. In each block, an equal number of trials consisted of a full match, a spatial mismatch, a functional mismatch, and a double mismatch. Thus, half of the trials required a match response, and half required a mismatch response.

The course of each trial was as follows: First, a fixation cross appeared in the middle of the screen for $300 \mathrm{~ms}$. After an interval of $400 \mathrm{~ms}$, the first frame was displayed for $800 \mathrm{~ms}$. In the instrument-first group, this frame showed a hand holding one of the eight instruments in one of the two orientations. In the target-first group, it showed one of the eight target objects in one of the two orientations. The second frame appeared immediately afterward with no interstimulus interval. For the instrument-first group, it now showed one of the eight target objects in both orientations, whereas for the target-first group it showed one of the eight instruments to

Table 1

Example Stimulus Matrix for the Different Combinations of Ticket, Ticket Canceller, Credit Card, and Credit Card Reader

\begin{tabular}{|c|c|c|c|c|c|}
\hline Plug & $\begin{array}{c}\text { Target object } \\
\text { orientation }\end{array}$ & $\begin{array}{c}\text { Credit card reader: } \\
\text { horizontal }\end{array}$ & $\begin{array}{c}\text { Credit card reader: } \\
\text { vertical }\end{array}$ & $\begin{array}{l}\text { Ticket canceller: } \\
\text { horizontal }\end{array}$ & $\begin{array}{l}\text { Ticket canceller: } \\
\text { vertical }\end{array}$ \\
\hline Credit card & Horizontal & Full match & Spatial mismatch & Functional mismatch & Double mismatch \\
\hline Credit card & Vertical & Spatial mismatch & Full match & Double mismatch & Functional mismatch \\
\hline Ticket & Horizontal & Functional mismatch & Double mismatch & Full match & Spatial mismatch \\
\hline Ticket & Vertical & Double mismatch & Functional mismatch & Spatial mismatch & Full match \\
\hline
\end{tabular}

Note. Each stimulus occurs once in each of the four conditions. Thus, reaction times in the four different conditions are based on exactly the same stimuli. 
be inserted in the two orientations. This frame remained on the screen until a response was given. The upper time limit for judging whether the spatial relation was appropriate by pressing one of two keys was $1,500 \mathrm{~ms}$. If the judgment was correct, the next trial started after an intertrial interval of 200 ms. If an error was committed, or the response was too slow, a short error message was displayed.

\section{Results}

Figure 2 shows the RTs (upper panel) and error rates (lower panel) in Experiment 1A. Only trials in which the participants delivered a correct judgment were included in the analysis of RTs. Trials in which the participants pressed the wrong button $(10 \%)$ or
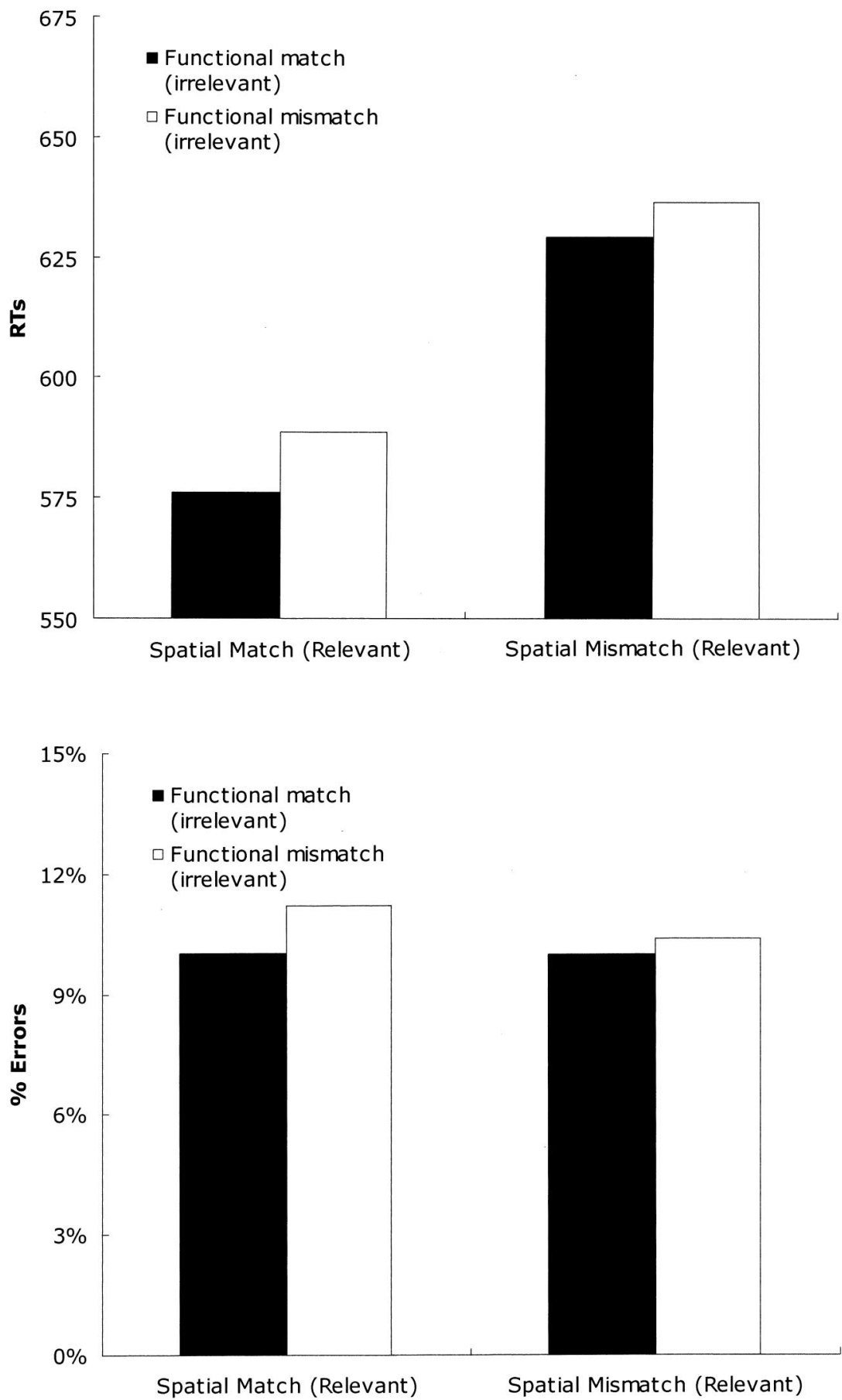

Figure 2. Reaction times (RTs; upper panel) and error rates (lower panel) in Experiment 1A (plugs, spatial task). The two bars on the left display the data for trials in which spatially matching actions had to be judged, and the two bars on the right display the data for trials in which spatially mismatching actions had to be judged. The black and white bars show irrelevant functional matches and mismatches, respectively. 
did not react in the given RT interval of $1,500 \mathrm{~ms}(4 \%)$ were excluded. The first 16 trials were considered training trials and were also excluded. The remaining reaction times were entered into a $2 \times 2 \times 2$ repeated-measurements analysis of variance (ANOVA) with the factors order of presentation (instrument first, target first), relevant relation (spatial match, spatial mismatch) and irrelevant relation (functional match, functional mismatch). The significance was tested at an alpha of .05 .

The ANOVA revealed a main effect for relevant relation, $F(1$, $30)=47.6, p<.01$. Actions were judged faster as being spatially matching $(M=582 \mathrm{~ms}, s=128 \mathrm{~ms})$ than mismatching $(M=633$ $\mathrm{ms}, s=122 \mathrm{~ms})$. There also was a main effect for irrelevant relation, $F(1,30)=19.07, p<.01$. Actions were judged slower when there was an irrelevant functional mismatch $(M=612 \mathrm{~ms}$, $s=124 \mathrm{~ms})$ than when there was none ( $M=602 \mathrm{~ms}, s=124 \mathrm{~ms})$. Order of presentation approached significance, $F(1,30)=3.33$, but did not interact with any other factor.

The error rates were entered into the same ANOVA. There were significant effects for neither relevant relation, $F(1,30)=.08$, nor order of presentation, $F(1,30)=1.46$. The main effect of irrelevant relation approached significance, $F(1,30)=3.24$.

\section{Discussion}

The spatial relation was judged faster when instrument and target object were functionally related, although the functional relation was task irrelevant and was to be ignored. Order of presentation did not modify the effects, although ratings of the stimuli showed that the associations from the target objects to the appropriate instruments were stronger than vice versa (see the Appendix); numerically the effect even pointed in the reverse direction. This finding therefore suggests that the appropriate action representations can only be accessed by combinations of instrument and target object but not by one of the two objects alone. Thus, for functionally related objects there was an action representation that also supplied information as to the action carried out with these objects, thereby facilitating the spatial task.

The lack of a difference between the two orders of presentation also counters arguments that the effects were due to priming of often co-occurring stimuli (i.e., participants had more often seen functionally related objects together than functionally unrelated objects). If this had been the case, the target objects should have primed the respective instruments to a stronger extent than vice versa. Additional evidence against such a view was provided by the finding that across actions, there was no correlation between the size of the effect of irrelevant functional mismatches and the ratings in terms of (a) familiarity and (b) the strength of the associations between instruments and target objects (see Table A1 in the Appendix).
In everyday action perception, and different from the present experiment, observers have no advance knowledge as to the action that is to be carried out. Experiment 1B investigated the effect of irrelevant functional mismatches in a more ecologically valid setup.

\section{Experiment 1B: Tools}

The present experiment investigated the influence of irrelevant functional mismatches in a less restricted action domain of tool use (e.g., see Figure 3). A variety of action types that differed in the spatial relations they required were presented at random. One half of the actions required identical orientations of instrument and target object to succeed (cleaning a plate with a sponge), whereas the other half required orthogonal orientations (cutting a piece of paper with a pair of scissors). The participants again were required to judge whether the orientation of the objects was appropriate for an action to be carried out with them. Because the different action types were presented at random, participants could no longer compare an observed spatial relation to an action type specified in the instruction. We hypothesized that participants would compensate for the lack of advance knowledge about the type of action to be judged by accessing the respective action representation by means of the functional relation. Thus, if the effects of irrelevant functional mismatches reflected the activation of action representations, they would be enhanced. Order of presentation was again varied between participants to investigate asymmetric influences of instruments and target objects on action comprehension.

\section{Method}

Participants. Thirty-two participants (16 men, 16 women), all students at the University of Munich, took part in the experiment. None of them had participated in Experiment 1A. They ranged in age from 20 to 35 years. All participants were right-handed and had normal or corrected-to-normal vision. They received payment for their participation. The same factors as in the previous experiment were counterbalanced.

Material and apparatus. The stimulus set consisted of 32 photographs. They obtained 10 visual degrees vertically and $7^{\circ}$ horizontally, given the viewing distance of $60 \mathrm{~cm}$. Sixteen pictures showed a hand holding one of the eight different tools (saw, gas pipe pliers, scissors, wire cutter, sponge, hole punch, rasp, or sandpaper) in two different orientations each (vertically or horizontally). The other half of the pictures showed the corresponding eight target objects (bough, pipe, piece of paper, wire, plate, stash of paper, cucumber, or a wooden timber), again, in both orientations. Out of these photographs, two-frame sequences were assembled. This was done analogous to the previous experiment.

For all of these sequences, ratings of (a) the familiarity of the actions, (b) the strength of the associations between instruments and target objects, and (c) the salience of the action effect were obtained (see the Appendix). The apparatus was the same as in Experiment 1A.

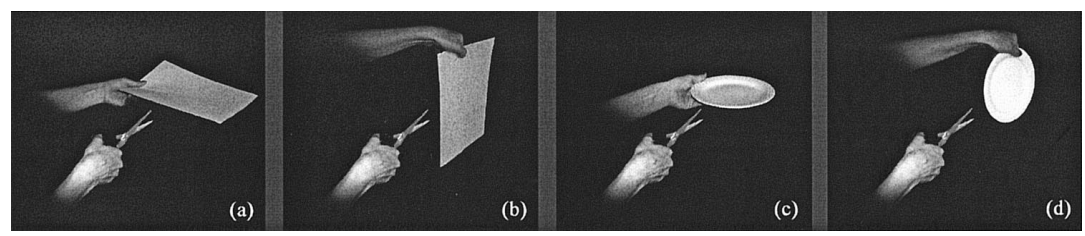

Figure 3. Examples for actions requiring orthogonal orientations of instrument and target object: (a) full match, (b) spatial mismatch, (c) functional mismatch, and (d) double mismatch. 
Procedure and design. Upon entering the lab, participants received a computer-based, written instruction. They were told that they would see one object being applied to another object and that their task was to judge whether the orientations of the instrument and the target object were appropriate to carry out an action. Again, they were also told to ignore whether the two objects usually were used together.

In all other respects, the procedure and design were the same as in the previous experiment. This implies that the actions requiring orthogonal and identical orientations of instruments and target objects were presented at random.

\section{Results}

Figure 4 shows the RTs (upper panel) and error rates (lower panel) in Experiment 1B. Only trials in which the participants had
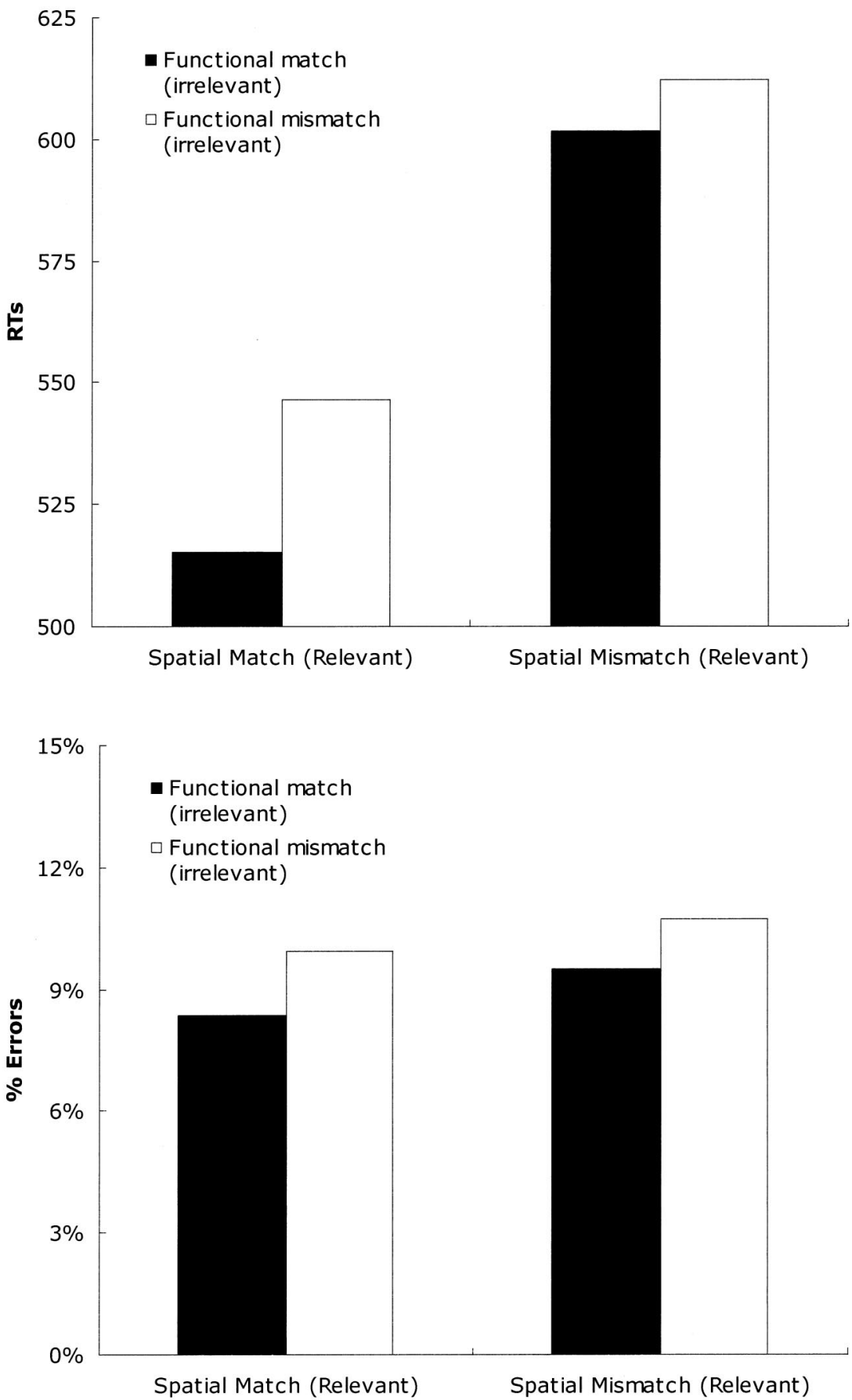

Figure 4. Reaction times (RTs; upper panel) and error rates (lower panel) in Experiment 1B (tools, functional task). The two bars on the left display the data for trials in which spatially matching actions had to be judged, and the two bars on the right display the data for trials in which spatially mismatching actions had to be judged. The black and white bars show irrelevant functional matches and mismatches, respectively. 
delivered a correct judgment were included in the analysis of RTs. Trials in which the participants pressed the wrong button (4\%) or did not react in time $(4 \%)$ were excluded. The first 16 trials were considered training trials and were also excluded. The remaining RTs were entered into a $2 \times 2 \times 2$ repeated-measurements ANOVA with the between-participants factor order of presentation (instrument first, target object first) and the within-participants factors relevant relation (spatial match, spatial mismatch) and irrelevant relation (functional match, functional mismatch). The significance was tested at an alpha of .05

There was no effect of order of presentation, $F(1,30)=.02$. The ANOVA revealed a main effect for relevant relation, $F(1,30)=$ $56.18, p<.01$. Participants were faster in judging spatially matching $(M=531 \mathrm{~ms}, s=135 \mathrm{~ms})$ than judging spatially mismatching actions $(M=607 \mathrm{~ms}, s=142 \mathrm{~ms})$. There also was a main effect of irrelevant relation, $F(1,30)=28.27, p<.01$, which was further qualified by an interaction of relevant relation and irrelevant relation, $F(1,30)=16.53, p<.01$. Post hoc Duncan tests showed that irrelevant functional mismatches slowed down the judgments of spatially matching actions (functional match, $M=515 \mathrm{~ms}, s=$ $129 \mathrm{~ms}$; functional mismatch, $M=547 \mathrm{~ms}, s=141 \mathrm{~ms} ; p<.01$ ) and, to a lesser extent, those of spatially mismatching actions (functional match, $M=602 \mathrm{~ms}, s=137 \mathrm{~ms}$; functional mismatch, $M=612 \mathrm{~ms}, s=148 \mathrm{~ms} ; p<.01)$. We also checked whether the results were affected by whether the actions required orthogonal or identical orientations of instrument and target object. However, no further significant effects were revealed when this factor was included in the ANOVA.

Numerically, the effect of irrelevant functional mismatches was larger than in the previous experiment. We investigated whether this difference was significant. The RTs of the two experiments were entered into a repeated measurements ANOVA with the between-participants factor experiment (plugs, tools) and the within-participants factors relevant relation (relevant relation is appropriate or not appropriate) and irrelevant relation (irrelevant functional relation is appropriate or not appropriate). The ANOVA revealed a significant interaction of irrelevant relation and experiment, $F(1,30)=5.92, p<.05$. Thus, the effect was stronger in Experiment 1B than in Experiment 1A.

Errors were entered into the same ANOVA. There was no main effect for order of presentation, $F(1,30)=3.09$, orientation, $F(1$, $30)=0.84$ or relevant relation, $F(1,30)=0.45$. However, a main effect for irrelevant relation was found, $F(1,30)=8.44, p<.01$. Irrelevant functional mismatches made the judgment of the spatial relation less reliable (functional match: $M=8.9 \%, s=8.3 \%$; functional mismatch: $M=10.3 \%, s=9.2 \%$ ).

\section{Discussion}

The effect of irrelevant functional mismatches reported in the previous experiment was replicated. Irrelevant functional mismatches slowed down the judgment of spatially matching and mismatching actions. Compared with the effect shown in Experiment $1 \mathrm{~A}$, the effect of the irrelevant functional relation was enhanced. This enhancement argues against the notion that the effects were due to priming of often co-occurring stimuli, because the actions in the tool domain were less familiar than those in the plugging domain and because the tools and instruments were less strongly associated with one another (see Figure A1 in the Appendix). Consistently, across actions, the effect of the irrelevant func- tional mismatches correlated neither (a) with ratings of the strength of the associations between the objects nor (b) with the familiarity of the actions (see Table A1 in the Appendix).

There was, however, across actions, a significant correlation between the saliency of the action goal and the RT difference between irrelevant functional matches and mismatches. This is consistent with our view that the action representations subserve the deriving of the goal of the action. The enhanced effect of irrelevant functional mismatches then suggests that participants used the functional relation to access the respective action representation in order to gain knowledge about the action to be carried out, to which, then, the given spatial relation was compared. This additional step was necessary because other than in the previous experiment, participants did not have advance knowledge about the action to be carried out in each trial.

\section{Experiment 2A: Plugs}

The first two experiments provided evidence that the functional relation between target objects and instruments is taken into account even when irrelevant and to be ignored. In the remaining two experiments, we investigated whether action representations also mediate performance in a functional task. Again, participants were tested for the domain of plugging actions first. Participants decided whether the two presented objects were usually applied to one another, irrespective of whether the spatial relation indicated that a plugging action was carried out. We predicted that irrelevant spatial mismatches would slow down the judgment of the functional relation. As in the previous experiments, the order of presentation was varied to investigate whether instruments predefine the spatial relation. If this were the case, larger effects of irrelevant spatial mismatches should be obtained when the instrument is presented in the first frame.

\section{Method}

Participants. Thirty-two participants (16 men, 16 women), all students at the University of Munich, took part in the experiment. None of them had participated in the previous experiments. They ranged in age from 20 to 35 years. All participants were right-handed and had normal or corrected-tonormal vision. They received payment for their participation. The same factors as in the previous experiments were counterbalanced.

Material and apparatus. The material and apparatus were identical to Experiment 1A.

Procedure and design. The procedure was identical to the previous two experiments, with one exception. In the written instruction to this experiment, the participants were told that their task was to judge whether the two objects were usually plugged into one another. In addition, they were asked to ignore whether the orientations of the two objects were appropriate to carry out a plugging action.

\section{Results}

Figure 5 shows the RTs (upper panel) and error rates (lower panel) in Experiment 2A. Only trials in which the participants had delivered a correct judgment were included in the analysis of RTs. Trials in which the participants pressed the wrong button $(6 \%)$ or did not react in the given interval of $1,500 \mathrm{~ms}(4 \%)$ were excluded. The first 16 trials were considered training trials and were also excluded. The remaining RTs were entered into a repeatedmeasurements ANOVA with the between-participants factors order of presentation (instrument first, target first), and the within- 

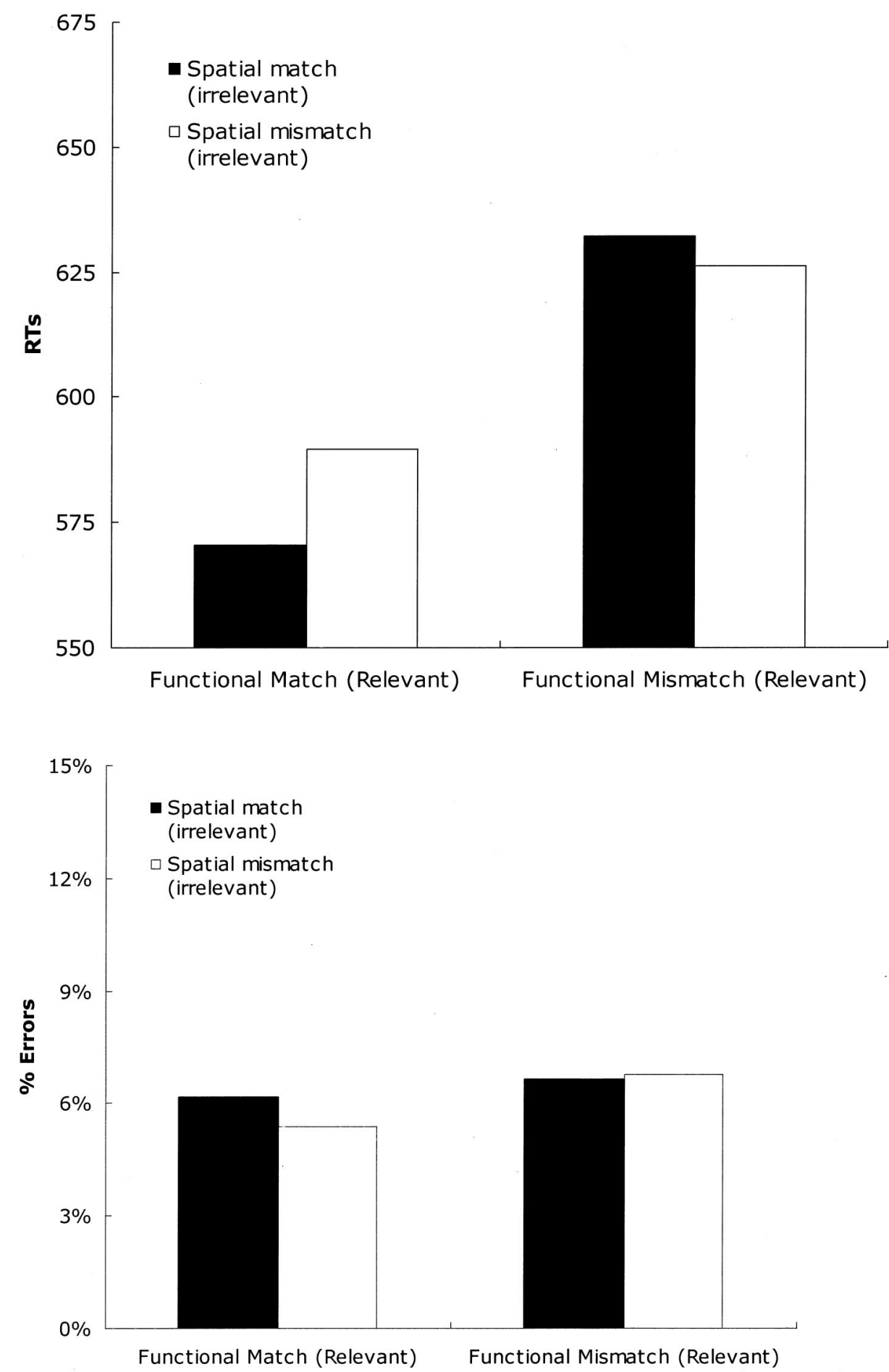

Figure 5. Reaction times (RTs; upper panel) and error rates (lower panel) in Experiment 2A (plugs, spatial task). The two bars on the left display the data for trials in which functionally matching actions had to be judged, and the two bars on the right display the data for trials in which functionally mismatching actions had to be judged. The black and white bars show irrelevant spatial matches and mismatches, respectively.

participants factors relevant relation (functional match, functional mismatch) and irrelevant relation (spatial match, spatial mismatch). The significance was tested at an alpha of .05.

There was no significant main effect for irrelevant relation, $F(1$, $30)=2.52$ and for order of presentation, $F(1,30)=3.70$. The
ANOVA revealed a main effect of relevant relation, $F(1,30)=$ 65.6, $p<.01$. Participants were faster reporting a match $(M=580$ $\mathrm{ms}, s=104 \mathrm{~ms})$ than reporting a mismatch $(M=629 \mathrm{~ms}, s=101$ $\mathrm{ms})$. There also was an interaction of relevant relation and irrelevant relation, $F(1,30)=9.74, p<.01$. Irrelevant spatial mis- 
matches slowed down the judgment of functional matches (spatial match: $M=571 \mathrm{~ms}, s=104 \mathrm{~ms}$; spatial mismatch: $M=590 \mathrm{~ms}$, $s=107 \mathrm{~ms}, p<.01)$. For functional mismatches, there was no such difference.

The error rates were entered into the same ANOVA (cf. Figure $5 \mathrm{~B})$. No main effects were obtained for relevant relation, $F(1$, $30)=0.22$, irrelevant relation, $F(1,30)=0.38$, and order of presentation, $F(1,30)=2.56$. There were no interactions between the factors. However, for functional matches, a numerical difference contrary to that observed in RTs was apparent. Participants made fewer errors when judging functional matches that were accompanied by a spatial mismatch $(M=6.1 \%, s=3.6 \%)$ than when there was none $(M=5.4 \%, s=3.7 \%)$. Although the effect for RTs was significant but that for error rates was not, it is critical to assess whether these inverse patterns of error rates and RTs mark a speed-accuracy trade-off. To test this, we computed the correlation between the differences in error rates and RTs between spatially matching and mismatching judgment. There was no evidence of a significant negative correlation $(r=.09)$. Thus, there was no speed-accuracy trade-off.

\section{Discussion}

Irrelevant spatial mismatches slowed down judgments of functionally matching actions. Again, across the different actions, the size of the difference did neither correlate with (a) the strength of the associations between instruments and target objects, and (b) the familiarity of the actions (see Table A1 in the Appendix). As such, it is unlikely that priming of often co-occurring stimuli was responsible for this result.

In contrast to irrelevant functional mismatches, irrelevant spatial mismatches had no effect on the judgment of functionally mismatching actions. One explanation for this finding might be that spatial mismatches created a faster decaying code than did functional mismatches. Because judgments of functional mismatches were always given later than those of functional matches, the code for spatial mismatches might already have disappeared. However, there was no correlation between the size of the effect for mismatching judgments and the delay of mismatching judgments that would support this explanation $(r=-.08)$.

The finding of irrelevant spatial mismatches affecting only the judgment of functional matches suggests that the processing of the spatial relation depends on an appropriate functional relation. This is consistent with the view that for functionally related objects there was no corresponding action representation. However, to interpret this effect with confidence, we attempted to replicate it within another action domain. Such a replication might also resolve a further problem of the present experiment. Although eight different combinations of instruments and target objects were presented to the participants, each depicted a plugging action. Thus, for each action, identical orientations of plug and target object were required. This being the case, the effect of irrelevant spatial mismatches could simply reflect a process of visual priming. An orientation perceived in the first stimulus might visually prime identical orientations in the second stimulus. In Experiment 2B we attempted to avoid this confound.

\section{Experiment 2B: Effect of Irrelevant Spatial Mismatches (Tools)}

The effect of irrelevant spatial mismatches was investigated within the domain of tool use. As described above, this domain included a variety of actions that required different spatial relations. One half of the actions required identical orientations of instrument and target object (e.g., cleaning a dish with a sponge). The other half required orthogonal orientations (e.g. cutting a piece of paper with a pair of scissors). For the latter class of actions, identical orientations actually constitute spatial mismatches. For these types of actions, the orientation-priming explanation predicts that inappropriate spatial relations speed up the responses, as instrument and target object would have identical orientations. If, however, the effect of irrelevant spatial mismatches is due to deriving the spatial relation in regard to possible actions, identical orientations should also impair the judgment of these types of actions. Again, the order of presentation was varied to test for asymmetric influences of instruments and target objects for the establishment of the spatial relation.

\section{Method}

Participants. Thirty-two participants (16 men, 16 women), all students at the University of Munich, took part in the experiment. None of them had participated in the previous experiments. They ranged in age from 20 to 35 years. All participants were right-handed and had normal or corrected-tonormal vision. They received payment for their participation. The same factors as in the previous experiments were counterbalanced.

Material and apparatus. The material and apparatus were identical to those of Experiment 1B.

Procedure and design. In the written instruction to this experiment, the participants were told that their task was to judge whether the two objects were usually applied to one another. They were asked to ignore whether the orientations of the two objects were appropriate for an action to be carried out. As in Experiment 1B, the actions requiring orthogonal and identical orientations were presented at random. In all other respects, the procedure and the design were identical to those of the previous experiment.

\section{Results}

Figure 6 shows the RTs (upper panels) and error rates (lower panels) in Experiment 2B. Only trials in which the participants had delivered a correct judgment were included in the analysis of RTs. Trials in which the participants pressed the wrong button $(5 \%)$ or did not react in time $(5 \%)$ were excluded. The first 16 trials were considered training trials and were also excluded. The remaining RTs were entered into a $2 \times 2 \times 2 \times 2$ repeated measurements ANOVA with the between-participants factor order of presentation (instrument first, target object first) and the within-participants factors relevant relation (functional match, functional mismatch), irrelevant relation (spatial match, spatial mismatch), and orientation (orthogonal orientations required, identical orientations required). The significance was tested at an alpha of .05.

There was a main effect of relevant relation, $F(1,30)=33.30$, $p<.01$. Once again, participants were faster in judging functional matches $(M=506 \mathrm{~ms}, s=128 \mathrm{~ms})$ than functional mismatches $(M=544 \mathrm{~ms}, s=136 \mathrm{~ms})$. No significant main effects were obtained for the factors irrelevant relation, $F(1,30)=1.45$, orientation, $F(1,30)=3.41$, and order of presentation, $F(1,30)=$ 0.15 . The ANOVA revealed, however, an interaction of relevant relation and orientation, $F(1,30)=4.41, p<.05$. For the actions 

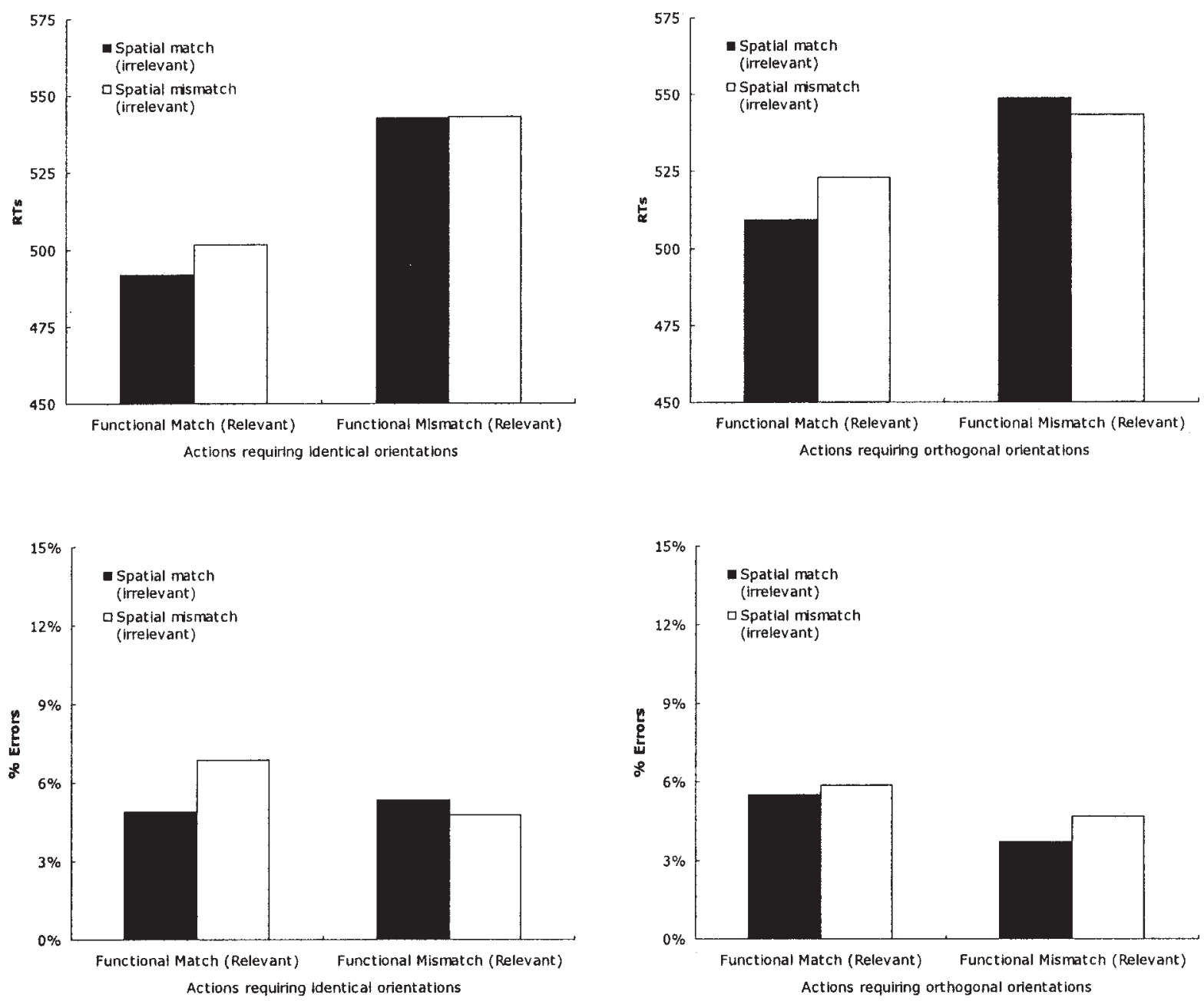

Figure 6. Reaction times (RTs; upper two panels) and error rates (lower two panels) of actions requiring identical (left panels) and orthogonal spatial relations (right panels) in Experiment 2B (tools, functional task). In each panel, the two bars on the left display the data for trials in which functionally matching actions had to be judged, and the two bars on the right display the data for trials in which functionally mismatching actions had to be judged. The black and white bars show irrelevant spatial matches and mismatches, respectively.

requiring identical orientations, there was a large difference between the judgment of matches $(M=497 \mathrm{~ms}, s=129 \mathrm{~ms})$ and mismatches $(M=543 \mathrm{~ms}, s=136 \mathrm{~ms})$. This difference was smaller for actions requiring orthogonal orientations (matching: $M=516 \mathrm{~ms}, s=132 \mathrm{~ms}$; mismatching: $M=546 \mathrm{~ms}, s=138$ $\mathrm{ms})$.

There was also an interaction of relevant relation and irrelevant relation, $F(1,30)=8.27, p<.01$. Post hoc Duncan tests show that an irrelevant spatial mismatch slowed down the judgment of functional matches ( $p<.005$; spatial match: $M=501 \mathrm{~ms}, s=128$ ms; spatial mismatch: $M=512 \mathrm{~ms}, s=131 \mathrm{~ms}$ ). For functional mismatches there was no such difference (spatial match: $M=546$ ms, $s=134 \mathrm{~ms}$; spatial mismatch: $M=543 \mathrm{~ms}, s=139 \mathrm{~ms}$ ). None of the effects was further qualified by orientation.

The errors were entered into the same ANOVA. No main effects were obtained for relevant relation, $F(1,30)=4.01$, irrelevant relation, $F(1,30)=3.06$, orientation, $F(1,30)=0.67$, and order of presentation, $F(1,30)=.16$. It revealed only a significant three-way interaction of relevant relation, irrelevant relation, and orientation, $F(1,30)=4.57, p<.05$. Only the error rates for functional matches requiring identical orientations were affected by an irrelevant spatial mismatch (spatial match: $M=4.9 \% \mathrm{~ms}$, $s=6.1 \%$; spatial mismatch: $M=6.8 \%$, ms, $s=7.5 \%$ ).

\section{Discussion}

The results of Experiment 2A were replicated. Judgments of functionally matching actions were faster when the irrelevant spatial relation was also matching. Because this difference was found for actions requiring identical and orthogonal spatial relations, it cannot be explained by assuming a process of visual priming. Rather, this finding is consistent with the view that the action representations mediating the judgment of the appropriate functional relation also include information about the actions usually carried out with these objects. As in the previous experiment irrelevant spatial mismatches only affected the judgment of func- 
tional matches. Again, there was no indication that the effect was due to the RT difference between match and mismatch judgments because the correlation between the size of the effect for mismatching judgments and the delay of mismatching judgments was positive $(r=.20)$. We therefore suggest that the processing of the spatial relation depends on an appropriate functional relation. For functionally unrelated objects, no such action representation exists, and therefore, the detection of a functional mismatch is not affected by irrelevant spatial mismatches.

Also note that, again, the effect of irrelevant spatial mismatches evoked by the different actions did not correlate with their familiarity nor did it correlate with the strength of the associations between instruments and target objects. The significant correlation with the saliency of the action goal, however, illustrates our interpretations that these action representations subserve the deriving of the ultimate goal of the action.

\section{General Discussion}

In four experiments, we investigated whether observers were able to selectively judge whether the spatial relation between an instrument and a target object was appropriate for an action to be carried out or whether the objects were functionally related. Both spatial and functional mismatches affected the judgment of the respective other relation even though the mismatches were completely irrelevant to the task and had to be ignored. Functional mismatches slowed down the identification of a spatial match and - to a lesser extent - the identification of a spatial mismatch. Irrelevant spatial mismatches also slowed down the identification of a functional match but did not slow down the identification of a spatial mismatch. Such interdependencies were predicted if (a) perceived actions were mapped onto the action representations an observer would rely on if he or she carried out the actions, and if (b) these representations incorporated information about which objects had to be used and which action had to be carried out for the goal of the action to be achieved.

Very similar results were obtained in a patient study by Riddoch and colleagues (Riddoch, Humphreys, Edwards, Baker, \& Wilson, 2003). The authors examined patients suffering from visual extinction who had difficulties in perceiving two objects at the same time. Visual extinction was, however, alleviated when the objects were both functionally related (e.g., pencil, ruler) and when they were presented in a spatial configuration suitable for an action to be carried out with them (e.g., pencil above ruler). The authors argued that action relations defined in both spatial and functional terms bound the objects together. Thus, the present findings can be seen as an extension of the findings of Riddoch et al. (2003) with healthy participants. In addition, our findings provide insights into how these representations are accessed during action perception. Each of the experiments suggested that an appropriate functional relation was a boundary condition for the activation of the integrated action representations.

In Experiment 1A, in which participants had to decide whether the spatial relation between two objects was appropriate for a plugging action, the judgments were facilitated if the two objects were functionally related. This effect cannot be attributed to priming of often co-occurring stimuli. In this domain, the target objects were more strongly associated to the instruments than vice versa (see the Appendix). If the effect were due to instruments priming the target objects and vice versa, the effect of irrelevant functional mismatches should have been larger when the target objects were presented first. However, no such difference was observed. $\mathrm{Nu}$ merically, the effect was even larger when instruments were presented first. Second, even when functionally unrelated, each of the single objects in a given trial afforded a plugging action (e.g., ticket, credit card reader). As such, functionally related objects activated plugging actions to a stronger extent than each object itself. This suggests that there was an action representation for functionally related objects that also specified the action with which these objects are applied to one another.

Converging evidence for this notion was provided by the comparison of Experiment 1A and 1B. In Experiment 1A, participants had advance knowledge that in every trial a plugging action was carried out, whereas in Experiment 1B, they did not have this knowledge because a variety of action types were presented at random. In Experiment 1B, the effect of irrelevant functional mismatches was enhanced. This suggests that participants accessed an action representation by means of functional information to compensate for the lack of advance knowledge. The enhancement also provides further evidence against priming accounts. A priming account would have predicted a smaller effect in Experiment $1 \mathrm{~B}$ because the actions in the domain of tool use were less familiar and the two objects involved were less strongly associated. Furthermore, the delay of irrelevant functional mismatches did not correlate with the familiarity of the actions nor did it correlate with the strength of the associations between the objects. However, it did correlate with the saliency of the action goal (see Table A1 in the Appendix). This finding cannot be explained by priming accounts, but it is consistent with our view that these representations subserve the deriving of the goal of a perceived action.

Another possibility that warrants consideration is that an appropriate functional relation might have informed participants about the parts of the objects relevant for the spatial task (e.g., in the plugging domain: the tip of the screwdriver and the slot of the screw) and directed their attention toward these parts. However, in the present paradigm, it was not necessary to localize the relevant parts because the stimuli were designed in such a way that the relevant parts were always presented at the same spatial positions. Moreover, if anything, the need to localize the relevant parts existed in the plug domain for target objects only, for which only the slot was rotated. In the tool domain and for instruments in the plugging domain, the rotations always affected the objects as a whole. If such a process was the source of the observed effects of irrelevant functional mismatches, (a) these effects should have been stronger in the plugging domain compared with the tool domain, and (b) there should have been an influence of the order of presentation in the plugging domain. However, neither of these effects was observed.

Performance in the functional task (Experiments $2 \mathrm{~A}$ and $2 \mathrm{~B}$ ) also suggested an important contribution of functional information to the activation of the action representations. Participants had to decide whether the two objects were usually applied to one another. They detected a functional match more easily when the spatial relation evoked the particular action usually carried out with these objects. Thus, an appropriate spatial relation provided additional activation to an action representation that was already accessed on the basis of functional information. This notion is confirmed by the finding that for functionally unrelated objectsfor which there should be no corresponding action representation-an irrelevant spatial mismatch did not lead to delayed RTs. 
This was the case even when each of the two objects afforded a plugging action in Experiment 2A. Thus, the effect of irrelevant spatial mismatches was brought about by knowledge about the particular action that was carried out with the given target object and the particular instrument but not by the actions associated with the particular objects themselves. This interpretation is complemented by the significant correlation between the delay of irrelevant spatial mismatches and the saliency of the action goals in the tool domain (see Table A1 in the Appendix). Although preliminary, this finding suggests that an action representation was accessed because the combination of objects was appropriate to produce a meaningful effect in the environment. An appropriate action derived from the spatial relation provided additional activation for this representation.

The strong influence of functional information on the activation of the action representations might be surprising in the light of theories claiming that the processes of the parietal-premotor pathway alone (which culminate in the firing of mirror neurons) are sufficient for action understanding. If, however, the parietalpremotor pathway is oblivious to the function of the elements, this claim has to be qualified. When actions involving instruments are perceived, the functional relation seems to provide a context in which the actions are judged. One possibility is that as soon as an object is identified in the temporal lobe, direct connections could provide the parietal cortex with information about the actions usually carried out with the observed object (e.g., Chao \& Martin, 2000; Johnson-Frey, 2004), thereby facilitating the spatial task. However, it is not clear whether the temporo-parietal connections supply the parietal system with the object parts relevant for action (Creem \& Proffitt, 2001; Goodale \& Humphrey, 1998) or with the actions themselves. For instance, Passingham and Toni (2001; see also Toni, Ramnani, Josephs, Ashburner, \& Passingham, 2001) have shown that the actions associated to stimuli are transferred to the parietal-premotor pathway via the prefrontal cortex but not directly from the temporal to the parietal lobes. Moreover, as discussed above, the effects of the irrelevant functional mismatches did not depend on the single objects involved. Rather, they depended on the relation between them. This was complemented by the finding of significant correlations between the RT costs for irrelevant functional mismatches and the saliency of the goal for different actions in the tool domain. As of yet, it has not been shown that the temporal cortex codes such relational or goal-related information.

It is also unclear how parieto-temporal connections would account for the effects of irrelevant spatial mismatches on functional judgments. Assuming that these connections are bidirectional, an action identified in parietal-premotor pathway might prime the objects usually used in this action, thereby facilitating the functional task. However, the parietal cortex (from which these connections originate) is usually not assumed to represent the type of action perceived. Such knowledge is available only later in the processing stream (i.e., the premotor cortex). In addition, parietotemporal connections cannot explain why irrelevant spatial mismatches impaired the judgments of functional matches but not the judgments of functional mismatches because the parietal-premotor pathway is functionally blind. Thus, objects associated with an action should be primed regardless of whether these objects are functionally related.

The results seem to be more consistent with the assumption that the observed interactions were mediated by the prefrontal cortex.
The prefrontal cortex is connected to both the temporal lobe and to the parietal-premotor pathway and is therefore ideally located to receive functional information about objects and about the type of action perceived. In addition, earlier research on action production suggests that the prefrontal cortex receives information from areas in the ventral stream about an object's identity and that it selects an appropriate action with respect to the actor's intention by biasing neurons in the premotor or parietal cortices (Arbib \& Bota, 2003; Rizzolatti \& Luppino, 2003). In action perception, the prefrontal cortex could fulfill a similar function. In such a model the action type and the objects are initially identified separately in the parietal-premotor pathway and the temporal lobe, respectively. The prefrontal cortex would then bind object-related and actionrelated aspects into an integrated action representation when the objects are functionally related. If such a role of the prefrontal cortex were confirmed in neuroimaging studies, one would have to conclude that the areas of the parietal-premotor pathway are not the only areas that show mirror properties. Rather, functional information about objects might also be a part of the representations that guide action production as well as action understanding.

A final question is whether the presentation of effectors is necessary to evoke the observed effects. This does not seem be the case. In the aforementioned study of Riddoch et al. (2003), effects of the spatial and functional relations were observed in the absence of effectors. This does not necessarily imply that the results have no implication for action comprehension. In fact, the authors argued that the patients were still "seeing the action" although no effectors were presented. This interpretation is consistent with findings that the mere presentation of objects suffices to activate the actions one could carry out with them (e.g., Chao \& Martin, 2000) and that actors conceive tools as if they were their effectors (e.g., Maravita, Husain, Clarke, \& Driver, 2001; Maravita, Spence, Kennetta, \& Driver, 2002). This is nicely illustrated by various Hollywood movies demonstrating that humans are perfectly capable of understanding actions even if the objects were handled by an "invisible man." One might therefore conclude that the action representations do not code the action itself but a goal state (defined in spatial and functional terms) that has to be achieved for the action to realize its intended effect. Given that there are reciprocal connections between such action representations and the goal of the action, accessing these representations might provide one avenue for the observer to truly understand a perceived action.

\section{References}

Arbib, M., \& Bota, N. (2003). Language evolution: Neural homologies and neuroinformatics. Neural Networks, 16, 1237-1260.

Brass, M., Bekkering, H., Wohlschläger, A., \& Prinz, W. (2000). Compatibility between observed and executed finger movements: Comparing symbolic, spatial and imitative cues. Brain and Cognition, 44, 124-143.

Bremmer, F., Duhamel, J.-R., \& Ben Hamed, S. (1996). Non-retinocentric coding of visual space in the macaque ventral intraparietal area (VIP). Abstracts of the Society for Neuroscience, 22, 666-668.

Buccino, G., Binkofski, F., Fink, G. R., Fadiga, L., Fogassi, L., Gallese, V., et al. (2001). Action observation activates premotor and parietal areas in a somatotopic manner: An fMRI study. European Journal of Neuroscience, 13, 400-404.

Chao, L. L., \& Martin, A. (2000). Representation of manipulable manmade objects in the dorsal stream. NeuroImage, 12, 478-484.

Craighero, L., Fadiga, L., Rizzolatti, G., \& Umiltà, C. (1999). Action for perception: A motor-visual attentional effect. Journal of Experimental Psychology: Human Perception and Performance, 25, 1673-1692. 
Creem, S. H., \& Proffitt, D. R. (2001). Grasping objects by their handles: A necessary interaction between cognition and action. Journal of Experimental Psychology: Human Perception and Performance, 1, 218-228.

Decety, J., \& Chaminade, T. (2003). When the self represents the other: A new cognitive neuroscience view on psychological identification. Consciousness \& Cognition, 12, 577-596.

Decety, J., Grèzes, J., Costes, N., Perani, D., Jeannerod, M., Procyk, E., et al. (1997). Brain activity during observation of actions: Influence of action content and subject's strategy. Brain, 120, 1763-1777.

DiPellegrino, G., Fadiga, L., Fogassi, L., Gallese, L., \& Rizzolatti, G. (1992). Understanding motor events: A neurophysiological study. Experimental Brain Research, 91, 176-189.

Gallese, L., Fadiga, L., Fogassi, L., \& Rizzolatti, G. (1996). Action recognition in the premotor cortex. Brain, 119, 593-609.

Goodale, M. A., \& Humphrey, G. K. (1998). The objects of action and perception. Cognition, 67, 181-207.

Hodges, J., Spatt, J., \& Patterson, K. (1999). "What" and "how": Evidence for the dissociation of object knowledge and mechanical problemsolving skills in the human brain. Proceedings of the National Academy of Sciences of the United States of America, Vol. 96, 9444-9448.

Hommel, B., Müsseler, J., Aschersleben, G., \& Prinz, W. (2001). The theory of event coding (TEC): A framework for perception and action planning. Behavioral and Brain Sciences, 24, 849-878.

Humphreys, G. W., \& Riddoch, M. J. (2001). Detection by action: Neuropsychological evidence for action-defined templates in search. Nature Neuroscience, 4, 84-88.

Iacoboni, M. (2005). Understanding others: Imitation, language, empathy. In S. Hurley \& N. Chater (Eds.), Perspectives on imitation: From cognitive neuroscience to social science. Cambridge, MA: MIT Press.

Jeannerod, M., Arbib, M. A., Rizzolatti, G., \& Sakata, H. (1995). Grasping objects: The cortical mechanisms of visuomotor transformation. Trends in Neuroscience, 18, 314-320.

Johnson, B. W., \& Hamm, J. P. (2000). High-density mapping in an N400 paradigm: Evidence for bilateral temporal lobe generators. Clinical Neurophysiology, 111, 532-545.

Johnson-Frey, S. C. (2004). The neural basis of complex tool use in humans. Trends in Cognitive Sciences, 8, 71-78.

Kilner, J. M., Paulignan, Y., \& Blakemore, S. J. (2003). An interference effect of observed biological movement on action. Current Biology, 13, 522-525.

Knoblich, G., \& Flach, R. (2003). Action identity. Consciousness and Cognition, 12, 620-632.

Kourtzi, Z., \& Kanwisher, N. (2000). Activation in human MT/MST by static images with implied motion. Journal of Cognitive Neuroscience 12, 48-55.

Kutas, M., \& Federmeier, K. D. (2000). Electrophysiology reveals semantic memory use in language comprehension. Trends in Cognitive Sciences, 4, 463-470.

Manthey, S., Schubotz, R. I., \& von Cramon, D. Y. (2003). Premotor cortex in observing erroneous action: An fMRI study. Cognitive Brain Research, 15, 296-307.

Maravita, A., Husain, M., Clarke, K., \& Driver, J. (2001). Reaching with a tool extends visual-tactile interactions into far space: Evidence from cross-modal extinction. Neuropsychologia, 39, 580-585.

Maravita, A., Spence, C., Kennetta, S., \& Driver, J. (2002). Tool-use changes multimodal spatial interactions between vision and touch in normal humans. Cognition, 83, B25-B34.

Martin, A., \& Chao, L. L. (2001). Semantic memory and the brain: Structure and processes. Current Opinion in Neurobiology, 11, 194-201.

Meltzoff, A. N., \& Decety, J. (2003). What imitation tells us about social cognition: A rapprochement between developmental psychology and cognitive neuroscience. Philosophical Transactions of the Royal Society of London, Series B, 358, 491-500.
Milner, A. D., \& Goodale, M. A. (1995). The visual brain in action. Oxford, United Kingdom: Oxford University Press.

Murata, A., Gallese, V., Luppino, G., Kaseda, M., \& Sakata, S. (2000). Selectivity for the shape, size, and orientation of objects for grasping in neurons of monkey parietal area AIP. Journal of Neurophysiology, 83, 2580-2601.

Nobre, A. C., \& McCarthy, G. (1995). Language-related field potentials in the anteriormedial temporal lobe: II. Effects of word type and semantic priming. Journal of Neuroscience, 15, 1090-1098.

Nowak, M. A., Plotkin, J. B., \& Jansen, V. A. A. (2000). The evolution of syntactic communication. Nature, 404, 495-498.

Ochipa, C., Rothi, L. J. G., \& Heilman, K. M. (1989). Ideational apraxia: A deficit in tool selection and use. Annuals of Neurology, 25, 190-193.

Oztop, E., \& Arbib, M. A. (2002). Schema design and implementation of the grasp-related mirror neuron system. Biological Cybernetics, 87, 116-140.

Passingham, R. E., \& Toni, I. (2001). Contrasting the dorsal and ventral visual systems: Guidance of movement versus decision making. Neuroimage, 14, 125-131.

Prinz, W. (2005). An ideomotor approach to imitation. In S. Hurley \& N. Chater (Eds.), Perspectives on imitation: From mirror neurons to memes. Cambridge, MA: MIT Press.

Riddoch, J. M., Humphreys, G. W., Edwards, S., Baker, T., \& Wilson, K. (2003). Seeing the action: Neurophysiological evidence for action-based effects on object selection. Nature Neuroscience, 6, 82-89.

Rizzolatti, G., Fadiga, L., Gallese, V., \& Fogassi, L. (1996). Premotor cortex and the recognition of motor actions. Cognitive Brain Research, 3, 131-141.

Rizzolatti, G., Fogassi, L., \& Gallese, V. (2000). Cortical mechanisms subserving object grasping and action recognition: A new view on the cortical motor functions. In M. S. Gazzaniga (Ed.), The new cognitive neurosciences (2nd ed., pp. 539-552). Cambridge, MA: MIT Press.

Rizzolatti, G., Fogassi, L., \& Gallese, V. (2001). Neurophysiological mechanisms underlying the understanding and imitation of action. $\mathrm{Na}$ ture Reviews Neuroscience, 2, 661-670.

Rizzolatti, G., \& Luppino, G. (2003). Grasping movements: Visuomotor transformations. In M. A. Arbib (Ed.), The handbook of brain theory and neural networks (2nd ed., pp. 501-504). Cambridge, MA: MIT Press.

Rizzolatti, G., Luppino, G., \& Matelli, M. (1998). The organization of the cortical motor system: New concepts. Electroencephalography and Clinical Neurophysiology, 106, 283-296.

Rossetti, Y., \& Pisella, L. (2002). Several 'vision for action' systems: A guide to dissociating and integrating dorsal and ventral functions. In W. Prinz \& B. Hommel (Eds.), Common mechanisms in perception and action: Attention and performance XIX (pp. 62-119). Oxford, United Kingdom: Oxford University Press.

Sakata, H., \& Taira, M. (1994). Parietal control of hand action. Current Opinion in Neurobiology, 4, 847-856.

Sitnikova, T., Kuperberg, G., \& Holcomb, P. J., (2003). Semantic integration in videos of real-world events: An electrophysiological investigation. Psychophysiology, 40, 160-164.

Stürmer, B., Aschersleben, G., \& Prinz, W. (2000). Correspondence effects with manual gestures and postures: A study of imitation. Journal of Experimental Psychology: Human Perception and Performance, 26, 1746-1759.

Toni, I., Ramnani, N., Josephs, O., Ashburner, J., \& Passingham, R. E. (2001). Learning arbitrary visuomotor associations: Temporal dynamic of brain activity. Neuroimage, 14, 1048-1057.

Tucker, M., \& Ellis, R. (1998). On the relations between seen objects and components of potential actions. Journal of Experimental Psychology: Human Perception and Performance, 24, 830-846.

Tucker, M., \& Ellis, R. (2001). The potentiation of grasp types during visual object recognition. Visual Cognition, 8, 769-800.

Ungerleider, L. G., \& Mishkin, M. (1982). Two cortical visual systems. In D. J. Ingle, M. A. Goodale, \& R. J. W. Mansfield (Eds.), Analysis of visual behaviour. Cambridge, MA: MIT Press. 


\section{Appendix}

\section{Ratings of the Stimuli}

Twenty-five participants who did not participate in any of the experiments completed questionnaires about the stimuli used in the present experiments. The questionnaire consisted of six pages and could be completed in less than $5 \mathrm{~min}$ time. It had three distinct parts. Each part contained the eight fully appropriate combinations of instruments and target object from each action domain used in the present experiments.

\section{Associations Between Instruments and Target Objects}

The reciprocal associations between instruments and target objects were assessed. Participants rated the actions in terms of how many instruments other than the depicted instrument could be applied to the depicted target object (very few, few, average, many, very many). The rationale was that the more other instruments could be applied to a given target object, the weaker the associations from the target object to the particular instrument should be. In a second step, participants also judged to how many other target objects a given instrument could be applied. Figure A1 (top panel) shows the results of the rating task.

A repeated-measures ANOVA with the factors domain (plugging, tool use) and type (instrument, target object) was conducted on the data. The results revealed significant main effects of domain, $F(1,24)=107.7, p<$ .01 , and type, $F(1,24)=10.0, p<.01$, and an interaction of the two factors, $F(1,24)=9.8, p<.01$. Post hoc Duncan tests show that in the plugging domain, instruments were more strongly associated to the target objects than the target objects were to the instruments (from target objects to instruments, $M=4.3, s=.62 \mathrm{~ms}$; from instruments to target objects, $M=3.6, s=.55 \mathrm{~ms} ; p<.01)$. For the tool domain, there was no such difference (from target objects to instruments, $M=2.5, s=.82 \mathrm{~ms}$; from instruments to target objects, $M=2.6, s=.71 \mathrm{~ms} ; p=.64$ ). In addition, in the plugging domain, the associations from instruments to target objects and the associations from target objects to instruments were stronger than in the domain of tools use (both $p \mathrm{~s}<.0001$ ).

\section{Familiarity With the Actions}

In the second part, the familiarity of the actions was assessed. The same actions as in the first part were presented. Participants indicated on a scale of five levels (very rarely, rarely, average, often, very often) how often they had observed the depicted actions and how often they had carried out these actions. Figure A1 (middle panel) shows the results of the rating task.

An ANOVA with the factors experience (observation, production) and domain (plugs, tools) was conducted on the data. The results revealed a main effect for experience, $F(1,24)=7.7, p<.05$, indicating that the actions were more often observed $(M=3.8, s=.81 \mathrm{~ms})$ than carried out $(M=3.6, s=.87 \mathrm{~ms})$. In addition, there was a main effect for domain, $F(1$, $24)=48.2, p<.01$, reflecting that the actions in the plug domain $(M=$ $4.1, s=.75 \mathrm{~ms})$ were more familiar than those in the tool domain $(M=$ $3.3, s=.79 \mathrm{~ms})$.

Figure A1. Results of the rating task. The upper panel shows the strength of the associations from instruments to target objects (white bars) and from target objects to instruments (black bars) for both action domains (plugs on the left, tools on the right). Higher ratings correspond to fewer alternative target objects and instruments and therefore indicate stronger associations. The middle panel shows the ratings of the familiarity of the actions. For both action domains, black bars reflect how often these actions have been observed, and white bars mark how often they had been carried out by the participants. The lower panel marks the saliency of the action goal in both action domains. In all panels, the error bars represent the standard error of the mean.

\section{Saliency of the Action Goal}

In the third part, the saliency of these goals of the actions was assessed. The same actions as in the first two parts were presented. Each of them was
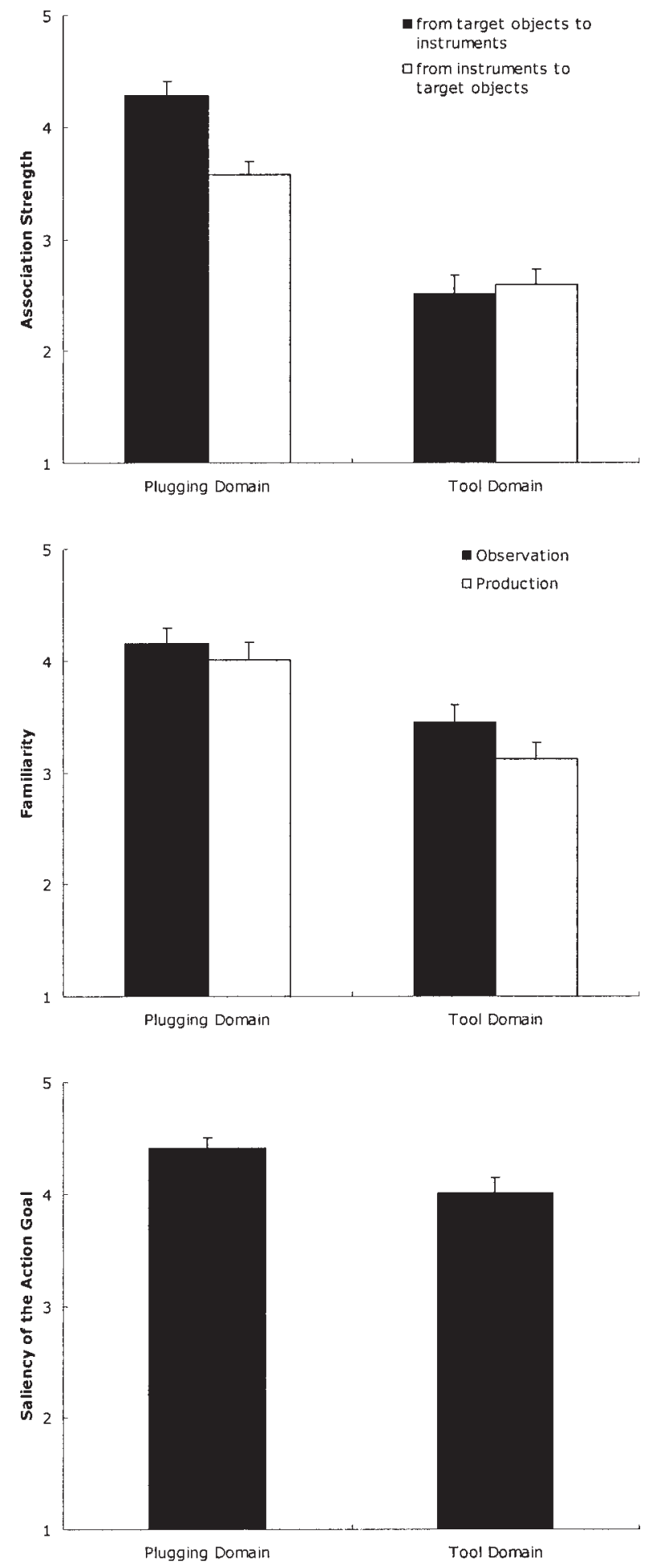
accompanied by a description of the goal to be achieved by the action (e.g., ticket, ticket canceller: "canceling a ticket"). The participants rated how apparent this goal was on a scale of five levels (very apparent, apparent, average, unapparent, very unapparent). Figure A1 shows the results of the rating task.

An ANOVA with the factor domain (plugs, tools) revealed a significant difference between the domain of plugging actions and that of tool use, $F(1,24)=6.0, p<.05$, reflecting that the action effects were less apparent in the domain of tool use $(M=4.1, s=.69 \mathrm{~ms})$ than in the domain of plugging actions $(M=4.4, s=.47 \mathrm{~ms})$.

\section{Correlations With the Observed Effects of Irrelevant Mismatches}

We also computed correlations between the different measures of the rating task for each of the actions with the actual size of the effect of the irrelevant mismatches as for each of the experiments, separately (see Table A1). To do this, the delay associated with the respective irrelevant mismatches was computed by subtracting the RTs for irrelevant mismatches from the RTs of the irrelevant matches in matching-judgments conditions for each of the eight stimuli in both orders of presentation (instruments and target objects). Thus, there were 16 different values of the delay of irrelevant mismatches for each experiment.

These delays were then correlated with the measures of the rating task, as follows. For correlations with the strength of the associations, the eight ratings of the target objects and the eight ratings of the instruments were assigned to the eight delay values from the two orders of presentation. Ratings of the associations from instruments to target objects were assigned to the delay values of the instrument-first conditions, and vice versa. For correlations with the two ratings of familiarity (observed and produced) and the salience of the effect, each value of the ratings was assigned to the corresponding two stimuli from both orders of presentation because
Table A1

Correlations of the Effects of Irrelevant Mismatches With the Measures of the Rating Task Across Stimuli

\begin{tabular}{lcccc}
\hline Experiment & $\begin{array}{c}\text { Familiarity: } \\
\text { observation }\end{array}$ & $\begin{array}{c}\text { Familiarity: } \\
\text { production }\end{array}$ & $\begin{array}{c}\text { Association } \\
\text { strength }\end{array}$ & $\begin{array}{c}\text { Saliency of the } \\
\text { action goal }\end{array}$ \\
\hline 1A: Plugs & -.21 & -.21 & .17 & -.36 \\
1B: Tools & .16 & .23 & .42 & $.62^{*}$ \\
2A: Plugs & .02 & -.18 & -.24 & .03 \\
2B: Tools & -.07 & -.15 & .15 & $.45^{*}$ \\
\hline
\end{tabular}

Note. In Experiments 1A and 1B space was relevant and function was irrelevant. In Experiment 2A and 2B function was relevant and space was irrelevant.

$* p<.05$, one-tailed.

whole actions were rated and not the single objects (e.g., the same rating of "canceling a ticket" was assigned to both the delay of irrelevant mismatches when the ticket canceller was presented first and the delay of irrelevant mismatches when the ticket was presented first).

The results showed that the delay associated with the irrelevant mismatches in the four experiments did not correlate with the strength of the associations between instruments and target objects, nor did it correlate with the familiarity of the actions (see Table A1). In the domain of tool use, however, the delay associated with irrelevant spatial and functional mismatches was correlated with the saliency of the action goal.

Received December 8, 2003

Revision received October 28, 2004

Accepted November 2, 2004

\section{E-Mail Notification of Your Latest Issue Online!}

Would you like to know when the next issue of your favorite APA journal will be available online? This service is now available to you. Sign up at http://watson.apa.org/ notify/ and you will be notified by e-mail when issues of interest to you become available! 